\title{
End to End Enteroanastomosis Following Enterectomy after Retrieval of an Intestinal Foreign Body in a Dobermann Dog
}

\author{
M. Gokulakrishnan ${ }^{1 *}$, G. Mohamed Ali ${ }^{1}$ and Ruth Jemima Arputharani Jeyaraj ${ }^{2}$ \\ ${ }^{1}$ Department of Clinics, Madras Veterinary College, Chennai Madras Veterinary College, \\ Tamil Nadu Veterinary and Animal Sciences University, Chennai-600 007 \\ ${ }^{2}$ Madras Veterinary College, Chennai \\ *Corresponding author
}

K e y w o r d s
Foreign body,
Enterectomy, End
to end,
Eneteroanastomosis,
Dobermann
Article Info
Accepted:
15 February 2020
Available Online:
10 March 2020

Keywords

Foreign body, Enterectomy, End to end,

Eneteroanastomosis, Dobermann

Article Info

15 February 2020

10 March 2020

\section{A B S T R A C T}

A one year old intact male Dobermann dog was brought to Small Animal Surgery Out Patient ward unit at Madras Veterinary College Teaching Hospital with the history of acute vomition and diarrhoea for the past four days. The animal was anorectic and severely dehydrated with a congested mucous membrane. A hard-painful irregular contoured moveable mass was observed on abdominal palpation. Lateral and ventrodorsal abdominal and thoracic radiographs were taken to rule out foreign body, which revealed a radiopaque irregularly shaped foreign body at the caudal intestine. An ultrasound examination disclosed the presence of a foreign body. A routine haematobiochemical profile was taken to ascertain organ health status. Marginal anaemia accompanied by increased ALP and BUN values were present. The animal was stabilised with fluid therapy in order to counteract dehydration and electrolyte imbalance. Midventral celiotomy was planned and performed following stabilisation. An enterotomy was done to remove the foreign body. Since the intestines cranial and caudal to the obstruction were devitalised, with the absence of peristalsis and mesenteric pulse, enterectomy followed by enteroanastomosis was performed. Standardised post-operative care and treatment were administered until the dog had an uneventful recovery.

\section{Introduction}

A one-year old intact male Dobermann dog was brought to Small Animal Surgery Out
Patient Ward Unit at Madras Veterinary College Teaching Hospital with the history of acute vomition and diarrhoea for the past four days. The animal was anorectic and severely 
dehydrated with a congested mucous membrane. A hard painful irregular contoured moveable mass was observed on abdominal palpation. Lateral and ventrodorsal abdominal and thoracic radiographs were taken to rule out foreign body, which revealed a radiopaque irregularly shaped foreign body at the caudal intestine. An ultrasound examination disclosed the presence of a foreign body. A routine haematobiochemical profile was taken to ascertain organ health status. Marginal anaemia accompanied by increased ALP and BUN values were present.

\section{Treatment}

The animal was treated with Ringer's Lactate, Pantoprazole, Cefotaxime, Trineurosol, Metronidazole and Ondansetron. After evaluation of the radiographs taken, it was determined to perform an emergency celiotomy as the radical remedy. The dog was premedicated with Diazepam @ $0.5 \mathrm{mg} / \mathrm{kg}$ body weight and Butorphanol @ $0.2 \mathrm{mg} / \mathrm{kg}$ body weight intravenously. Anaesthesia was induced with propofol @ 4mg/kg body weight intravenously. Following induction, intubation was performed with ID cuffed endotracheal tube. The animal was placed in dorsal recumbency and surgical draping was done. A linear incision was made on the linea alba through the skin. The portion of the intestine containing the foreign body was identified and exteriorised from the abdominal cavity. This segment of intestine was isolated from the abdominal cavity by packing with moist laparotomy sponges. The foreign body was retrieved by incising the healthy tissue proximal to the obstruction on the antimesenteric side. The segment of intestine was evaluated subjectively and was found to lack peristalsis and mesenteric pulse. As the tissues surrounding the foreign body were devitalised, the mesenteric blood vessels were doubly ligated and non-crushing intestinal forceps were used to outline the segment of intestine to be resected. Enterectomy was performed followed by enteroanastomosis. First the mesenteric side was sutured using PGA 3-0 in a simple interrupted pattern. This was continued up to the antimesenteric side by simple interrupted pattern placing the sutures $2 \mathrm{~cm}$ from the edge and $3 \mathrm{~cm}$ apart. Once this was completed 10 $\mathrm{ml}$ of sterile normal saline was infused into the anastomosed site to assess any leakage. To ensure vitality of the anastomosed intestines was adequate, the presence of peristalsis was also observed for. The surgical site was sutured in a cross mattress pattern using polyamide 2-0 suture material. Bandaging was done and antibiotics were given. The owner was advised to give oral antibiotics and to apply an E-collar. Th pet had an uneventful recovery without any complications. The animal was stabilised with fluid therapy in order to counteract dehydration and electrolyte imbalance. Midventral celiotomy was planned and performed following stabilisation. An enterotomy was done to remove the foreign body. Since the intestines cranial and caudal to the obstruction were devitalised, with the absence of peristalsis and mesenteric pulse, enterectomy followed by enteroanastomosis was performed. Standardised post-operative care and treatment were administered until the dog had an uneventful recovery.

Alimentary tract obstruction was one of the most common ailments noticed in dogs (Orshe and Rosin, 1993). The incidence rate of GIT obstruction is very high in young male dogs due to their voracious, indiscriminative feeding habits and playful nature (Kumar et al., 2000). All along the length of gastrointestinal tract foreign bodies causes obstruction but jejunum is the most common location (Hayes, 2009). Some small, sharp foreign bodies, such as pins, sewing needles, and fish hooks that are found in asymptomatic animals may be treated conservatively and 
they may pass uneventfully, because of contact between mucosa of intestine and foreign body results in local dilation of intestine called as mural withdrawal reflex (Guilford et al., 1996). Gastrointestinal foreign bodies may cause complete or partial obstruction. The size of the foreign body determines whether obstruction is partial or complete as observed in the present acse which was a sone of irregular contour. Lifethreatening complications caused by fluid and electrolyte imbalances, hypovolemia, and toxemia may be associated with intestinal foreign bodies (Papazoglou et al., 2001). Surgical interventions related to the treatment of small intestinal obstruction represents approximately $0.5-1$ percent of all surgical procedures in dogs (Crha et al., 2008).

Intestinal resection and anastomosis is commonly performed in veterinary patients for treatment of intestinal foreign bodies, intestinal perforation, devitalized tissue secondary to vascular compromise, intestinal neoplasia, or intussusception and in patients that require revision of prior intestinal surgery A variety of surgical techniques for intestinal anastomosis have been described and analysed (Ellision, 1990). Currently, both end-to-end sutured anastomoses and FEESAs are commonly accepted techniques. Various suture materials and patterns have been examined in the context of intestinal anastomoses (Capak et al., 2001). Singlelayer appositional closures are preferred to inverting or everting patterns, as submucosal apposition has been shown to promote primary healing of the intestinal wall, whereas inverted or everted closures require secondintention healing and can increase the risk of luminal stenosis or anastomosis site leakage, respectively. Several potential risk factors have been proposed for anastomotic dehiscence in veterinary patients, including hypoalbuminemia, preoperative peritonitis, presence of a foreign body, intraoperative hypotension, preexisting inflammatory bowel disease, and involvement of large intestine (Weisman et al., 1999). Intestinal healing is a complex process. Inflammatory responses anywhere within the bowel cause an increase in collagenase activity throughout the gastrointestinal tract. This collagenase activity decreases the collagen content of the submucosa, which is the holding layer for intestinal apposition. Collagen content reaches a nadir during the lag phase of healing, approximately 3 days after surgery, and the decreased collagen concentration weakens the tissue and delays healing. Intestinal enterotomy and anastomosis sites are therefore most susceptible to dehiscence during the lag phase (Toyomasu et al., 2010).
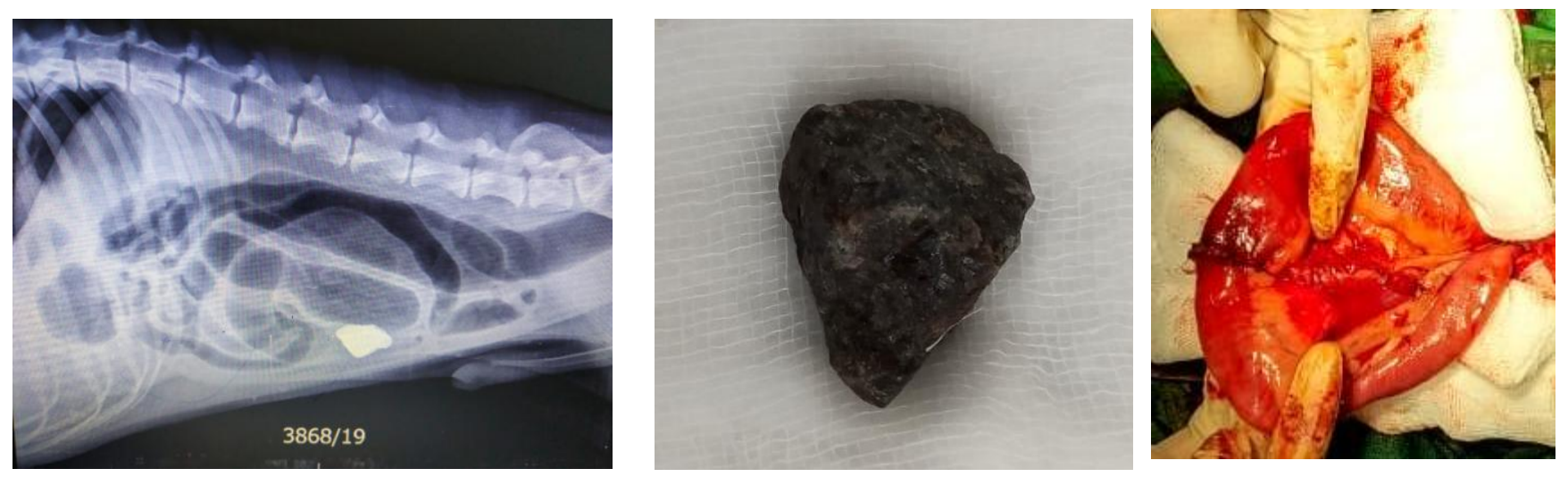
References

Capak, D., Simpraga, M. and Maticic, D. (2001). Incidence of foreign bodyinduced ileus in dogs. Berl Munch Tierarztl Wochenschr. 114:290-296.

Crha, M., Lorenzova, J., Urbanova, L., Fitchel, T. And Necas, A. (2008). Effect of preoperative mortality in dogs with small bowel obstruction. Acta. Veterinaria. Brno., 77:257-261.

Ellision, G.W. (1990). Enterotomy in current technique in small animal surgery. edited by MjosephBojrab, 3rd Saunders company, Philadelphia: 249.

Guilford, W.G. and Strombeck, D.R. (1996). Intestinal obstruction, pseudoobstruction, and foreign bodies, in Guilford, W.G., Center, S.A., Strombeck, D.R., et al., (eds). Strombeck's Small Animal Gastroenterology. Philadelphia, WB Saunders, 3rd ed: 487-502.

Hayes, G. (2009). Gastrointestinal foreign bodies in dogs and cats: a retrospective study of 208 cases. Journal of Small Animal Practice. 50:576-583.

Kumar, D.D., Ameerjan, K. and David, W.A.
(2000). Gasrto intestinal tract obstruction in dogs. I. J. Ver. Sur., 21:43-44.

Orshe, R.J. and Rosin, E. (1993). Small intestine, in Slatter DH Textbook of Small Animal Surgery, Philadelphia, WB Saunders. 2nd ed: 593-612.

Papazoglou, L. G., Patsikas, M. N. and Rallis, T. (2003). Intestinal foreign bodies in dogs and cats. Compendium of Continuing Education for the Practicing Veterinarian. 25: 830-843.

Toyomasu Y, Mochiki E, Ando $\mathrm{H}$, et al., 2010. Comparison of postoperative motility in hand-sewn end-toend anastomosis and functional end-to-end anastomosis: an experimental study in conscious dogs. Dig Dis Sci; 55: 24892497. [PubMed: 19915979].

Weisman, D.L., Smeak, D.D., Birchard, S.J. and Zweigart, S.L. (1999). Comparison of a continuous suture pattern with a simple interrupted pattern for enteric closure in dogs and cats: 83 cases (1991- 1997). J.A.V.M.A., 214:15071510.

\section{How to cite this article:}

Gokulakrishnan, M., G. Mohamed Ali and Ruth Jemima Arputharani Jeyaraj. 2020. End to End Enteroanastomosis Following Enterectomy after Retrieval of an Intestinal Foreign Body in a Dobermann Dog. Int.J.Curr.Microbiol.App.Sci. 9(03): 1916-1919.

doi: https://doi.org/10.20546/ijcmas.2020.903.222 